TRANS · núm. 25.2021

MISCELÁNEA $\cdot 265-279$

LITERARIA

En este trabajo hemos procedido a analizar los títulos de los cien capítulos que conforman el original y dos traducciones de la obra Viaje al Oeste en español. Mediante la construcción de un corpus con dichos textos analizamos las principales características y diferencias existentes entre ellos (en particular, a nivel sintáctico, semántico y léxico/terminológico), al igual que los distintos modos de verter el libro original y los diferentes recursos que emplean los traductores en los textos meta. De igual modo, constatamos la dificultad de verter al castellano una obra escrita en chino mandarín y la pérdida de información relevante en el proceso de traslación, debido a la dificultad de mantener el estilo, la rima, plasmar los aspectos interculturales en castellano y traducir correctamente las unidades terminológicas de las religiones orientales.

PALABRAS CLAVE: Viaje al Oeste, traducción literaria, corpus, títulos, chino-español.

\title{
Ante los títulos de los capítulos en las novelas clásicas chinas: análisis y comparación de las traducciones chino-español de Viaje al Oeste
}

\author{
TIAN MI \\ Rodrigo Muñoz Cabrera \\ Universidad de Nankai
}

\section{Facing Chapter Titles in Chinese Classical Novels: an analysis and a comparison of the Chinese-Spanish translations of Journey to the West}

In this research, we have studied the headings of the one hundred chapters of Journey to the West, and two of their translations into Spanish. By building up a corpus with these texts, we analyzed the main features and the differences among them -in particular, at the syntactic, semantic, and lexical/terminological levels-, as well as the different ways in which professionals translated the original text, and the resources they used to carry out their task. Likewise, we confirm the difficulty of translating a book written in Mandarin Chinese into Castilian Spanish, and the loss of relevant information during the translation process, due to the difficulty of keeping the style, the rhythm, of capturing the cross-cultural aspects in Spanish, and of correctly translating the terminological items of eastern religions.

KEY WORDS: Journey to the West, literary translation, corpus, titles, Chinese-Spanish.

\footnotetext{
* Este artículo forma parte de los resultados del proyecto de humanidades y ciencias sociales titulado «Estudios basados en corpus sobre las traducciones en español de Viaje al Oeste», financiado por el Fondo para Jóvenes Investigadores del Ministerio de Educación de la República Popular China (No: 18YJC740064).
} 


\section{INTRODUCCIÓN}

Las novelas clásicas chinas de las dinastías Ming (1368-1644) y Qing (1636-1912), que son fruto del trabajo de los artistas que narraban oralmente sucesos históricos y épicos en la dinastía Song (960-1279) (Zheng, 2012: 11), han conservado y desarrollado unas características particulares, entre las que cabe destacar como más representativa la forma en que los eruditos titularon cada capítulo de las novelas clásicas chinas.

Dichos epígrafes, además de facilitar a dichos narradores la memorización del argumento, resultaban indispensablemente para recopilar estas crónicas, que eran relativamente independientes entre sí, pero que podían conformar un conjunto basándose en un hilo conductor. De este modo, y gracias al esfuerzo de los literatos chinos, los textos que nos interesan adquirieron unas peculiaridades fonológicas, sintácticas, léxicas y pragmáticas a las que incorporaron sus gustos estéticos personales. Su forma se configuró al concluir el periodo central de la dinastía Ming y no cesó de reproducirse hasta finales de la dinastía Qing. Finalmente, dicha práctica se suprimió de las principales corrientes de creaciones literarias por ser considerada como anticuada después de la Revolución en el ámbito de la novela propuesta por Liang Qichao en noviembre de 1902 (Liu, 2018: 78).

«The practice of book title translation exhibits a fair amount of variability across time, space and genres» (Viezzi, 2011: 183). Los misioneros occidentales de los siglos XVIII y xIX que viajaron a China escribieron libros en mandarín o tradujeron a dicha lengua obras de la literatura occidental, imitando la redacción de los títulos de capítulos que aparecían en las novelas clásicas chinas para acercarse más a los lectores meta. De igual modo, los primeros traductores chinos también domesticaron los encabezamientos en obras oc- cidentales para que sus traslaciones pudieran adaptarse mejor al gusto local (Li, 2012: 438459). Posteriormente esta metodología fue desestimada por los nuevos eruditos chinos, entre los cuales, cabe destacar a Lin Shu (1852-1924), literato y traductor consagrado que trasladó, en colaboración con otros traductores, más de ciento ochenta obras de varios idiomas occidentales, incluida la primera versión de Don Quijote.

A pesar de que la forma de redactar los títulos de capítulos de las novelas clásicas chinas ya se ha convertido en una práctica en desuso (excepto en ciertas novelas de ficción modernas del género de las artes marciales), este hábito forma parte del acervo de la literatura china y muestra de una manera intensiva la estética de los literatos chinos de antaño. Debido a sus particularidades expresivas y su ubicación (al preceder el texto en cada capítulo), se trata del primer obstáculo con el que se encuentran los traductores en cuanto intentan verter las novelas clásicas chinas a otras lenguas.

En este artículo, pretendemos, en primer lugar, analizar los títulos de capítulos de las novelas clásicas chinas, tomando como ejemplo los de Viaje al Oeste, los cuales, según Li, representan la madurez de dicha práctica (2012: 190). En segundo lugar (y con el propósito de extraer los datos básicos para nuestro análisis), construimos un minicorpus paralelo, del estilo de los "bilingual comparable corpora" (Laviosa, 2002: 36), constituido por los títulos de capítulos originales y sus dos traslaciones completas.

Durante la compilación del mencionado corpus, utilizamos la herramienta NLPIRParser, diseñada y desarrollada en la Universidad de Beijing para distinguir y anotar gramaticalmente las palabras en mandarín. Como en dicha lengua los caracteres no se separan mediante un espacio, ello puede causar problemas al emplear los textos sin tratar en otras herramientas de análi- 


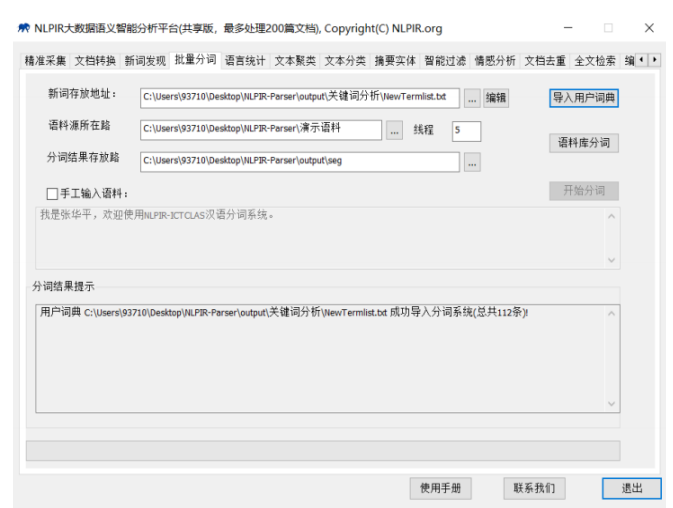

IMAGEN I. La ventanilla de trabajo de NLPIRParser

sis. De igual modo, y a causa de la abundancia de nombres propios y términos budistas y taoístas en el texto (además de las diferencias sintácticas y léxicas existentes en las obras clásicas y las contemporáneas), la detección de palabras y la adición de etiquetas automáticas requiere de un mayor esmero y corrección manual. Una vez digitalizados los tres textos, empleamos la herramienta WordSmith 7.0 para identificar las singularidades más representativas. Asimismo, hicimos uso del programa accesible por Internet, SketchEngine, para anotar gramaticalmente las palabras en los textos meta.

Nuestro propósito es, por una parte, aplicar el método de análisis basado en corpus en los estudios de traducción de chino al español y por otra, la comparación de los dos conjuntos de traducciones de los títulos de capítulos de dicha novela a niveles sintáctico y léxico. El motivo de dicha elección estriba en que es en los títulos donde se concentran con gran brevedad aspectos gramaticales, pragmáticos y estéticos particulares de las novelas clásicas chinas extensas. Debido a que se trata de un aspecto lingüístico y cultural que no ha sido debidamente analizado a día de hoy, hemos considerado oportuno descubrir las
DCH_titles__tagged - 记本本

文件 $(\mathrm{F})$ 编辑 $(\mathrm{E})$ 格式 $(\mathrm{O})$ 查看 $(\mathrm{V})$ 帮助 $(H)$

灵 $<a>$ 根 $<n>$ 育 $<\mathrm{vg}>$ 孕 $<n g>$ 源流 $<n>$ 出 $<\mathrm{vf}>.<w j>$ 心性 $<n>$ 修 $<\mathrm{v}>$ 持 $<\mathrm{v}>$ 大道 $<n>$ 生 $\langle\mathrm{v}\rangle$. $<\mathrm{wj}>$

悟 $<\mathrm{vg}>$ 彻 $<\mathrm{vg}>$ 菩提 $<\mathrm{n}>$ 真 $<\mathrm{d}>$ 妙 $<\mathrm{a}>$ 理 $\langle\mathrm{n}>.<\mathrm{wj}>$ 断 $<\mathrm{v}>$ 魔 $<\mathrm{n}>$ 归 $<\mathrm{v}>$ 本 $<\mathrm{rz}>$ 合 $\langle v\rangle$ 元 $\langle q\rangle$ 神 $\langle n>.<w j>$

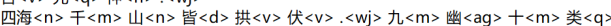
尽 $<$ vi $>$ 除名 $<$ vi $>,<w j>$

官 $<n>$ 封 $<v>$ 頍马 $<n>$ 心 $<n>$ 何 $<r y>$ 足 $<a>.<w j>$ 名 $<q>$ 注 $<v>$ 齐天 $<n>$ 意 $<n>$

末 $<\mathrm{d}>$ 宁 $<\mathrm{ag}>$. $<w j>$

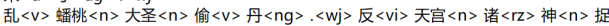
$\langle\mathrm{V}\rangle$ 怪 $\langle\mathrm{n}\rangle$. $<\mathrm{wj}>$

观音 $\langle n>$ 赴 $\langle v>$ 会 $\langle n>$ 问 $\langle v>$ 原因 $\langle n>$. $<w j>$ 小圣 $\langle n>$ 施威 $\langle v>$ 降 $\langle v>$ 大圣 $\langle n>$

$.<w j>$
八卦炉 $<n>$ 中 $<f>$ 逃 $\langle v>$ 大圣 $<n>.<w j>$ 五行山 $\langle n>$ 下 $<s>$ 定 $\langle v>$ 心猿 $<n>.<w j>$

我 $\langle\mathrm{rr}\rangle$ 佛 $\langle\mathrm{n}\rangle$ 造 $\langle\mathrm{v}\rangle$ 经 $\langle\mathrm{n}\rangle$ 传 $\langle\mathrm{v}\rangle$ 极乐 $\langle\mathrm{n}\rangle .\langle\mathrm{wj}\rangle$ 观音 $\langle\mathrm{n}\rangle$ 奉 $\langle\mathrm{v}\rangle$ 旨 $\langle\mathrm{ng}\rangle$ 上 $<\mathrm{f}\rangle$ 长安 $<\mathrm{n}>,\langle w \mathrm{wj}>$

陈光䒜 $<n>$ 赴任 $<v i>$ 逢 $<v>$ 灾 $<n>.<w j>$ 江流 $<n r>$ 僧 $<n>$ 复仇 $<v n>$ 报 $<n>$ 本 $\langle\mathrm{rz}\rangle,\langle w j\rangle$

老 $<a>$ 龙王 $<n>$ 拙计 $<n>$ 犯 $<v>$ 天条 $<n>.<w j>>>$ 魏 $<n r 1>$ 丞相 $<n>$ 遗书 $<n>$ 托 $<v>$ 冥 $<n g>$ 吏 $<n g>$. $<w j>$

游 $<\mathrm{v}>$ 地府 $<n>$ 太宗 $<n>$ 还魂 $<\mathrm{vi}>,<w j>$ 进 $<\mathrm{vf}>$ 瓜果 $<n>$ 刘全 $\langle n>$ 续 $<\mathrm{v}>$ 配 $<n>$ < $<$ wj $>$

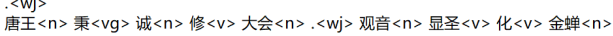

$<$ wj $>$

陷 $<\mathrm{vi}>$ 虎穴 $<n>$ 金星 $<n>$ 解 $<\mathrm{V}>$ 厄 $<n>$, $<w j>$ 双叉岭 $<n>$ 伯钦 $<n r 2>$ 留 $<v>$ 僧 $\langle$ ng $>,\langle w j\rangle$

心猿 $<n>$ 归 $<v>$ 正 $<n>$, $\langle w j>$ 六 $<m>$ 贼 $<n>$ 无 $\langle v>$ 踪 $<n g>,\langle w j>$ 蛇盘山 $\langle n>$ 诸 $<r z>$ 神 $\langle n>$ 暗 $\langle d>$ 佑 $\langle v>.<w j>$ 鹰愁涧 $\langle n>$ 意马 $\langle n>$ 收缰 $<v>$

.$<$ wj $>$
观音院

$<$ wi $>$

\section{IMAGEN 2. Vista parcial del texto automáticamente} etiquetado y manualmente corregido

técnicas adoptadas por los traductores a la hora de verter dichos encabezamientos. Estas técnicas pueden servir de referencia tanto para futuras prácticas de traducción de las obras clásicas chinas al español como para solucionar problemas traductológicos de naturaleza análoga.

\section{CARACTERÍSTICAS GENERALES DE LOS TÍ- TULOS DE CAPÍTULOS DE VIAJE AL OESTE}

Viaje al Oeste es una de las obras clásicas más conocidas de China. Trata del viaje de un grupo de peregrinos formado por el monje Tripitaka, un caballo-dragón y tres discípulos: un mono poderoso, un cerdo y un espíritu-bonzo. Se dirigen hacia al Oeste con el propósito de llevar sutras originales (textos sagrados del budismo) a China para difundirlos en su tierra natal y, durante el trayecto, tendrán que afrontar ochenta y una dificultades impuestas, principalmente, por malos espíritus o monstruos. La historia está basada en 
268 el recorrido realizado por Tripitaka durante la dinastía Tang (618-907 d.C.) y se transmitía boca a boca, incorporando, de ese modo, la imaginación creadora del pueblo chino. Finalmente, la narración se recopiló y se elaboró, supuestamente, por el literato Wu Cheng'en en la dinastía Ming (1368-1644), aunque aún faltan pruebas concluyentes que puedan justificar su autoría con toda certeza (Mi y Muñoz, 2020: 288-289).

$\mathrm{Al}$ igual que sus formas narrativas representativas, divididas en 100 capítulos, los títulos de estos también contienen peculiaridades. Cada encabezamiento lo conforman dos frases con estructuras sintácticas simétricas y se construyen con el mismo número de caracteres (un $3 \%$ de frases de cuatro caracteres, un $9 \%$ de cinco, un $76 \%$ de siete y un $12 \%$ de ocho), conformando así un paralelismo cada uno de ellos. Las palabras de las mismas categorías gramaticales que aparecen en las frases anteriores coinciden, en la mayoría de los casos, con las de las posteriores de cada capítulo, siguiendo el mismo orden de las primeras. De igual modo, y en cuanto se refiere a los nombres propios, estos también presentan una simetría semántica. Por ejemplo, si en la primera frase se muestran topónimos, estos pueden encontrarse también en la segunda.

Ejemplo 1) Frases de cuatro caracteres: (TO, capítulo 14) 心猿归正

Transcripción en pinyin: Xinyuan gui zheng

Ejemplo 2) Frases de cinco caracteres: (TO, capítulo 23) 三藏不忘本

Transcripción en pinyin: Sanzang bu wang ben

Ejemplo 3) Frases de ocho caracteres: (TO, capítulo 19) 云栈洞悟空收八戒 Transcripción en pinyin: Yuzhaidong Wukong shou Baijie
Estas disposiciones simétricas compuestas por unidades semánticas crean unidades rítmicas en cada título separadas con una pausa similar a las cesuras en los versos compuestos. Un $58 \%$ de estos está compuesto por dos frases de siete caracteres con la pausa ubicada detrás de los primeros cuatro caracteres de cada una de ellas, formando un paralelismo de «4+3/4+3», mientras que un $18 \%$ de este tipo posee una estructura «3+4/3+4». Cabe también precisar que las pausas rítmicas en las oraciones han sido marcadas mediante una barra oblicua («/»).

Ejemplo 4) Frases de siete caracteres, modelo «4+3/4+3»: (TO, capítulo 3) 四海千 山/皆拱伏

Transcripción en pinyin: Sihai qianshan/jie gongfu

Ejemplo 5) Frases de siete caracteres, modelo «3+4/3+4»: (TO, capítulo 11) 猪八戒/ 义激猴王

Transcripción en pinyin: Zhu Bajie/yi ji houwang

Así mismo, la combinación de los tonos que posee cada carácter forma una métrica singular similar a los pareados chinos. Además, y debido a la falta de conjugación temporal de los verbos en mandarín, todos los títulos se exhiben ante los lectores sin marcadores de tiempo precisos, lo que permite acercar la historia al lector con una mayor facilidad; el único indicio con el que se puede deducir el orden cronológico de los sucesos es mediante la posición sintáctica.

Con respecto al contenido de los títulos, se resume en ellos la trama central de cada capítulo mediante estas dos frases, constituyendo una prolepsis con la que se revela el desenlace antes de que el lector inicie la lectura. Igualmente se incluye una variedad de términos procedentes del budismo y del taoísmo en los títulos, los cua- 
les no solo presentan la temática, sino que también determinan los papeles de cada personaje, desvelándose, de ese modo, las relaciones entre unos y otros.

Su carácter exótico y oriental, a más del empleo de recursos lingüísticos singulares, junto con la brevedad de los títulos (en contraste con la larga extensión de la obra) dificulta sobremanera su traslado. Tomando en cuenta diferencias narrativas tales como la simplificación del nombre de los capítulos en las novelas modernas españolas, los títulos descriptivos de Viaje al Oeste recuerdan más a los de La Celestina o Don Quijote de la Mancha.

\section{COMPARACIÓN DE LAS CARACTERÍSTICAS GENERALES DE LAS TRADUCCIONES DE LOS TÍTULOS DE VIAJE AL OESTE}

Hoy en día se pueden encontrar en el mundo hispanohablante, traducciones parciales o por capítulos, historietas, adaptaciones novelísticas y cinematográficos de Viaje al Oeste (Ku, 2019: 55 56), además de dos versiones completas de dicha novela: la llevada a cabo por Enrique P. Gatón e Imelda Huang-Wang en 1992 y la segunda, publicada en 2010 y traducida indirectamente del francés al chino por María Lecea y Carlos Trigoso Sánchez. Considerando que estas dos translaciones completas conservan al máximo las particularidades de los títulos de capítulo de la Novela, construimos un corpus paralelo constituido por estas dos translaciones y el texto original, en base al cual se analizan y se comparan las traducciones de los títulos de los cien capítulos. Con el propósito de facilitar el análisis, denominaremos, a partir de este momento, «TO» a la obra original en chino, mientras que la vertida y publicada en castellano en 2010 será «TM1» y la de 1992 «TM2».
Veamos seguidamente el título del capítulo 1 y sus dos traducciones a modo de ejemplo. Marcamos en el título en chino las pausas rítmicas en oraciones con «/» y las que aparecen entre oraciones, con «//», mostrando su transcripción en pinyin debajo del texto original:

\section{Ejemplo 6) TO (Capítulo 1): 灵根/育孕/源 流/出//心性/修持/大道/生//}

Transcripción en pinyin: Linggen/yuyun/ yuanliu/chu//Xinxing/xiuchi/dadao/sheng//

TM1: De una piedra maravillosa surge la vida; El ser venido al mundo, aspirando a la propia perfección, alcanza el Gran Dao

TM2: CUANTO EXISTE TIENE SU ORIGEN EN LA RAÍZ DIVINA. EL TAO SURGE DIRECTAMENTE DE LA FUENTE MISMA DE LA MORALIDAD

Los títulos de los capítulos originales no disponen de signos de puntuación debido a la ausencia de este sistema ortográfico en la escritura de la Antigua China. Grosso modo, la versión TM1 marca cada capítulo mediante dos oraciones separadas con un punto y coma y pone en mayúscula la primera letra de cada capítulo, al igual que algunas letras iniciales de las palabras cuando su naturaleza lo requiere. Asimismo, se deja en mayúscula la primera letra de las palabras situadas detrás de un punto y coma. En contraposición con TM1, todos los títulos de TM2 se imprimen con las letras en mayúscula conforme a las normas ortográficas de castellano, estando compuestas por dos oraciones separadas por un punto.

Por medio de las herramientas Wordsmith 7.0 y SketchEngine, obtuvimos las características generales de los encabezamientos de cada versión, las cuales se muestran en la siguiente tabla: 
Tabla 1. Características generales de los títulos de los capítulos en Viaje al Oeste

\begin{tabular}{cccccc}
\hline Versión & Palabras & Formas & $\begin{array}{c}\text { Promedio } \\
\text { de formas/palabras }\end{array}$ & $\begin{array}{c}\text { No de palabras lle- } \\
\text { nas/No de palabras }\end{array}$ & $\begin{array}{c}\text { Promedio de longitud } \\
\text { de oración/palabra }\end{array}$ \\
\hline TO & 948 & 568 & 59,92 & $98 \%$ & 4,74 \\
\hline TM1 & 2084 & 727 & 41,35 & $52.6 \%$ & 10,42 \\
\hline TM2 & 2332 & 799 & 41,20 & $54.2 \%$ & 11,66 \\
\hline
\end{tabular}

A resultas de estos datos (Tabla 1), entre TM1 y TM2, el valor del promedio estándar de formas/ palabras (STTR en inglés) de la primera es ligeramente superior que en la segunda. Ello puede indicar que TM1 muestra una mayor variedad de vocablos. Asimismo, el promedio de longitud de los títulos en TM1 es alrededor de 2,2 veces superior que en el TO, mientras que en el TM2 es casi 2,5 veces mayor. Es evidente que los autores de TM2 suelen construir oraciones más largas que los de TM1.

Pasemos a examinar los datos de los porcentajes de palabras llenas y vacías en cada versión. Tomando en cuenta la peculiaridad en lo que se refiere a la clasificación de los géneros de palabras en mandarín, consideraremos a los adverbios como parte integrante del grupo de palabras llenas (Wang, 2014: 14). Según los datos que aparecen en la Tabla 1, la intensificación de información que nos ofrece el texto original es mucho más alta que la de sus dos traducciones y, entre TM1 y TM2, la última tiene un valor superior.

Si consultamos el listado de frecuencia de palabras de estas tres versiones (Tabla 2), podemos descubrir que tanto en la TM1 como en la TM2 la mayoría de las once primeras palabras más recurrentes son artículos, preposiciones y conjunciones que se catalogan como palabras vacías (las cuales no se muestran en la Tabla 2 por motivos de espacio). Por el contrario, en TO aparecen pa- labras llenas destacando, entre ellas, los nombres de los protagonistas incluidos tres distintos alias del Mono, e incluso un verbo (volver). Ello suele indicar, en la mayoría de los casos, una transformación de los personajes (en humano o en cualquier otro ser u objeto), al ser este uno de los poderes que poseen.

Por otro lado, el empleo de conjunciones (ausentes por completo en el texto original) indica que la transformación de frases simples a compuestas es notable. De la misma manera, el uso masivo de artículos explica parcialmente el destacable incremento de voces en las translaciones. Esto también indica un alto porcentaje de aparición de sustantivos en los dos textos meta. En ellas destacan las altas frecuencias de aparición de los nombres de los protagonistas, aunque en ambas la denominación «木母» (literalmente, Madre de Madera, que es el apelativo taoísta del Cerdo) no posee tanta visibilidad en comparación con el TO, debido a la omisión de este en ambas versiones.

Asimismo, las palabras 《真》 y 《正» (que significan literal y sucesivamente, auténtico y ver$\mathrm{dad}$ ) son términos budistas y taoístas y también préstamos del lenguaje secular chino utilizados para explicar conceptos abstractos y religiosos. Debido a ello, carecen de calcos en español y es difícil su representación con tan solo una palabra en la lengua meta. 
Tabla 2. Listado de frecuencia de palabras llenas de los títulos de capítulos

\begin{tabular}{clll}
\hline Número & TO & TM1 & TM2 \\
\hline 1 & 心猿 (MONO-ALMA) & Demonio (16) & Mente (12) \\
\hline 2 & 魔 (DEMONIO) & Wukong (17) & Mono (17) \\
\hline 3 & 归 (VOLVER) & Sun (18) & Montaña (21) \\
\hline 4 & 僧 (MONJE) & Gran (19) & Gran (22) \\
\hline 5 & 真 (AUTÉNTICO) & Monje (20) & Monstruo (23) \\
\hline 6 & 正 (VERDAD) & Mono-Alma (21) & Sabio (24) \\
\hline 8 & 大 (GRAN) & Demonios (22) & Rey (25) \\
\hline 9 & 大圣 (GRAN SABIO) & Es (23) & Peregrino (26) \\
\hline 10 & 怪 (MONSTRUO) & Rey (24) $(27)$ \\
\hline 11 & 孙行者 (SUN EL NOVICIO) & Guanyin (27) & Es (28) \\
\hline
\end{tabular}

Adicionalmente, debido a la naturaleza del idioma chino, que no muestra el espacio, el tiempo y la lógica por medio de marcadores gramaticales y conectores, el hilo temporal y las conexiones de las acciones se captan gracias a la colocación lineal de cada uno de los elementos. Esto añade dificultades a la comprensión del título si no se conoce previamente el contenido de cada capítulo. Se observa, por una parte, la aplicación de una serie de métodos gramaticales en ambas traducciones (tales como la conjugación temporal de los verbos o el complemento circunstancial) y, por otra, las grandes diferencias que se aprecian con frecuencia entre las dos versiones.

Frente a la notable diferencia entre el chino y el español (unida a las particularidades sintácticas que poseen los títulos de capítulos en las novelas clásicas chinas), hemos de preguntarnos si el proceso de traslación se puede cumplir con la máxima equivalencia dinámica que define Nida (2017), o por la que el traductor formula «un título que funcione en la cultura terminal» y al mismo tiempo respeta «con máxima fidelidad la intención del autor original» (Nord, 1990: 154). Para un lector chino resulta sencillo apreciar intuitivamente los títulos en forma de pareado con ritmo y métrica, al igual que (en no pocas ocasiones) su rima. Se trata de factores que son difíciles (si no imposibles) de recuperar o transferir en el texto meta. De igual modo, los términos budistas y taoístas desvelan connotaciones de valor y de religión existentes en la novela, aspecto poco familiar para lectores hispanohablantes; de ahí que ello pueda colocar a los traductores ante el dilema de la alternativa entre la estrategia de domesticación o de extranjerización, propuestas por Venuti (1995), o la de traducción libre o literal, dependiendo del isomorfismo en- 
272 tre las culturas, la formación de los traductores, la expectativa de ellos de los lectores meta, etc.

Teniendo en cuenta los problemas a los que se enfrentan los traductores, compararemos en los niveles sintáctico y léxico las dos versiones de los cien títulos de los capítulos de la novela Viaje al Oeste para llevar a cabo un análisis descriptivo y determinar los mejores métodos adoptados teniendo en consideración las características propias del TO.

\section{ANÁLISIS SINTÁCTICO Y ESTRATEGIAS TRA- DUCTOLÓGICAS}

\section{Paralelismo sintáctico}

En comparación con el español, las palabras en mandarín no tienen que someterse a una estricta regla de concordancia sintáctica. Además, cabe puntualizar que los verbos no se conjugan, de ahí que se suela disfrutar de más libertad a la hora de su ubicación dentro del discurso. Dicha particularidad se destaca, sobre todo, en los títulos de capítulos de las novelas clásicas, donde los autores intentan crear una simetría sintáctica y léxica para producir un efecto poético (Li, 2012: 10). Ello, por una parte, explica la estabilidad del número de palabras de cada oración en chino y, por otra, supone una gran dificultad para los traductores en el caso de que intenten interpretar y reproducir un equilibrio similar en la lengua meta.

Sin embargo, podemos comprobar que en esta novela los títulos conformados por dos oraciones simples de cuatro o cinco caracteres, en la mayoría de los casos, disponen de la misma estructura $(\mathrm{S}+\mathrm{V}+\mathrm{O})$. Como se aprecia en el ejemplo 2 , el primer carácter de cada oración se refiere al mono y al monje respectivamente, recurriéndose al uso de la metonimia. El segundo es un adjetivo que se coloca detrás del sustantivo, lo que es más común en las obras clásicas que en las mo- dernas de China; el resto constituye el predicado conforme a la estructura $\mathrm{V}+\mathrm{O}$. Resulta evidente que los traductores de ambas versiones son conscientes de recuperar cierta simetría sintáctica al traducir, puesto que la estructura $\mathrm{S}+\mathrm{V}+\mathrm{O}$ es «el orden básico o predominante» en español (Hernando Cuadrado, 2005: 163); de esta manera, no reviste una gran dificultad mantenerla en el texto meta. Por otro lado, los autores de TM1 acuden más al método de la perífrasis para aclarar a los lectores a quiénes se refieren el «espíritu» y el «tao» que aparecen en TM2.

Ejemplo 7) TO (Capítulo 56): 神狂/S诛/ $\mathrm{V}$ 草 寇/O// 道昧/S放/V心猿/O//

Transcripción en pinyin: Shenkuang/zhu/ caokou//Daomei/fang/xinyuan//

TM1: Sun Wukong, furioso, mata a unos salteadores de caminos; El monje Tang, ofuscado, despide al Mono-Alma

TM2: FUERA DE SÍ, EL ESPÍRITU ACABA CON LOS BANDIDOS. SUMIDO EN CONFUSIÓN, EL TAO TERMINA EXPULSANDO AL MONO DE LA MENTE

Ejemplo 8) TO (Capítulo 1): 观音院/僧/谋/ 宝贝//黑风山/怪/窃/袈裟//

Transcripción en pinyin: Guanyinyuan/seng/ mou/baobei//Heifengshan/guai/qie/jiasha//

TM1: El superior del Monasterio Guanyin piensa apoderarse del tesoro; El monstruo de la Montaña del Viento Negro roba la kasaya

TM2: LOS MONJES DEL MONASTERIO DE KWANG-ING TRATAN DE APODERARSE DEL TESORO. UN MONSTRUO ROBA LA TÚNICA EN LA MONTAÑA DEL VIENTO NEGRO 
La simetría sintáctica entre las dos estrofas de los títulos puede servir como un método de interpretación y una norma de referencia en lo que se refiere a la traslación. En el ejemplo 3, los primeros tres caracteres en mandarín son topónimos que se utilizan como adjetivos para determinar la procedencia de los personajes; el cuarto carácter de cada frase es el sujeto, mientras que el quinto es el verbo y los dos últimos forman objetos; por lo tanto, la estructura esencial del dístico también consiste en la $\mathrm{S}+\mathrm{V}+\mathrm{O}$. En la versión TM2, la traducción de la segunda frase supone un error, porque «la Montaña del Viento Negro» no constituye un complemento circunstancial de lugar, sino un adjetivo para indicar el origen del monstruo, con una función similar a la de «Monasterio Guanyin».

\section{Serialización verbal}

El resto de los encabezamientos que contienen siete caracteres presenta una diversidad de manifestaciones sintácticas, siendo una de las más representativas la serialización verbal. Así se denomina el fenómeno en que las frases contienen dos o más verbos que conjuntamente describen un evento; entre dichos verbos no hay conjunciones, pero sí que comparten unas características gramaticales comunes (Baker, 1989). En los títulos de los capítulos del Viaje al Oeste abunda este tipo de oración.

Ejemplo 9) TO: (Capítulo 8) 我佛/S造/V1 经/传/V2极乐//观音/s奉/V3旨/上/V4长安//

Transcripción en pinyin: Wofo/zaojing/chuan jilei// Guanyin/fengzhi/shang chang'an//

TM1: Buda crea las escrituras sagradas y difunde la beatitud suprema; Guanyin recibe la indicación de ir a Chang'an
TM2: PARA LA OBTENCIÓN DE LA FELICI-

DAD SUPREMA EL BUDA SOBERANO HA

CREADO LAS ESCRITURAS. KUANG-YIN RECIBE LA ORDEN DE DIRIGIRSE HACIA CHANG'AN

Ejemplo 10) TO: (Capítulo 9) 陈光芯/S赴/ $\mathrm{V} 1$ 任/逢/V2灾//江流僧/S复/V3仇/报/V4 本//

Transcripción en pinyin: Chen Guangrui/ furen/fengzai//Jiangliuseng/fuchou/baoben//

TM1: Chen Guangrui sufre una desgracia en su camino; El monje Traído por el Río venga el crimen cometido

TM2: AL IR A TOMAR POSESIÓN DE SU CARGO, CHEN KUANG-JUI SE TOPA CON LA DESGRACIA. AL SER VENGADOS, LOS PADRES RECIBEN EL PREMIO DEBIDO A SUS DESVELOS

En el ejemplo 4, V1 y V3 se anteponen a V2 y a V4 respectivamente según su sucesión temporal. Los traductores de TM1 modifican la primera oración simple en chino a una compuesta empleando la conjunción «y». En TM2, se sustituye el V2 con una construcción preposicional para indicar la finalidad de la acción y reproducen el hilo temporal del texto origen mediante la conjugación del tiempo verbal. En ambas versiones, se transforma el V4 a una construcción preposicional para determinar el $\mathrm{O} 3$.

En el ejemplo 5, se posicionan V1 y V3 antes de V2 y V4 con base a su relación lógica causa/efecto. En TM1, se remplaza la estructura V2+O2 por una construcción preposicional para indicar el lugar de la acción de V1, mientras que simplifican la segunda oración eliminando la parte V4+O4. En TM2, se transforma el V1+O1 a una subordinación adverbial que indica la circunstancia temporal del V2 que se coloca en la oración principal. En la segunda, los traductores cambian el 
Tabla 3. Número de oraciones en forma pasiva en TM1 y TM2

\begin{tabular}{cccc}
\hline Versión & $\begin{array}{c}\text { No de oraciones en forma } \\
\text { pasiva perifrástica }\end{array}$ & $\begin{array}{c}\text { No de oraciones en forma } \\
\text { pasiva refleja }\end{array}$ & Total \\
\hline TM1 & 13 & 15 & 28 \\
\hline TM2 & 9 & 2 & 11 \\
\hline
\end{tabular}

sujeto de V3 y V4, modificando, de esta manera, y por completo, el significado original.

La transformación de oraciones con serialización de verbos a oraciones compuestas contribuye a los altos porcentajes del uso de la conjunción «y» y la combinación «al», que aparecen en el listado de frecuencia de palabras. Asimismo, en comparación con TM2, la tendencia a no traducir por completo el contenido que se muestra en TM1 en estos ejemplos, puede explicar parcialmente la razón del valor menor del promedio de longitud de oración en ella, hecho que se observa en la Tabla 1.

\section{Omisión, abstracción o empleo de metonimia en el sujeto}

Según el listado de frecuencia de palabras, existe un alto índice en el uso de la forma «se» en ambas traducciones. Entre las múltiples funciones que desempeña, su empleo como componente de las pasivas reflejas nos llama la atención. En contraposición con el inglés o francés, la forma pasiva no es muy frecuente en español (Garcia Yebra, 1997: 348). Además, esta en chino suele expresar sufrimiento o un hecho sucedido contra nuestra voluntad (Wang, 2014: 88). Al igual que en castellano, se prefiere la construcción activa y, ante la pasiva perifrástica, siempre es recomendable la pasiva refleja (Gili Gaya, 1961: 122-124). En la actualidad, existen dos tipos de oraciones pasivas en chino: la primera se forma con el mar- cador «被》 (bèi) o palabra similar y la segunda sin marcadores (Wang, 2014: 93-99).

Después de consultar el original, podemos constatar que se mantiene la estructura pasiva en español mediante la utilización de la forma pasiva perifrástica. No obstante, las pocas oraciones pasivas existentes en el texto en mandarín no nos explican el motivo de la abundancia de «se» en los textos meta. Sea como fuere, en el resto de las traslaciones no es difícil encontrar otras oraciones con dicha peculiaridad.

En el TO solo hemos identificado 6 oraciones pasivas y, como se muestra arriba, en ambos textos meta aparece con mucha más profusión la voz pasiva que en la obra original. En comparación con TM2, los traductores de TM1 recurren más al uso de esta en su traslado. Si volvemos a examinar todas las oraciones en el TO, podemos descubrir que ello se debe al empleo de la omisión, la abstracción o el uso de la metonimia en el sujeto del texto original; de ahí que, en vez de correr el riesgo de intuir y recuperar el sujeto según el contexto, los traductores prefieran acudir a la voz pasiva para evitar cometer errores.

Tomemos la versión de la primera oración del título del capítulo 55 como ejemplo:

Ejemplo 11) TO (parte del Capítulo 55): 色 邪/淫戏/唐三藏//

Transcripción en pinyin: Sexie/yinxi/Tang Sanzang// 
TM1: Tang Sanzang es atacado por la tentación de la carne y el libertinaje;

TM2: LA FORMA MALVADA HACE PROPOSICIONES LASCIVAS A TRIPITAKA TANG.

En el original se usa la forma activa, al igual que en TM2 que, además, mantiene el mismo orden sintáctico que en el texto origen. No obstante, los traductores de TM1 prefieren utilizar el recurso de la voz pasiva para anteponer el sujeto paciente. Desde el ámbito de la psicología lingüística, TM2 destaca más «la forma malvada», mientras que TM1 pone más peso en «Tang Sanzang», posponiendo, de esta manera, el foco semántico original del TO. Esto puede deberse a que el autor hace uso de un nombre metonímico (色邪, sèxié, que, literalmente, significa la causa que tienta a la gente al libertinaje) para referirse a un mal espíritu de escorpión metamorfoseado en una hermosa mujer que quiere seducir al monje. Los traductores de TM1 transfieren a los lectores meta la connotación de los caracteres que constituyen el nombre, mientras que los de TM2 lo sustituyen en base a la prosodia semántica negativa que ofrece la descripción del personaje.

\section{ANÁLISIS LÉXICO Y SUS ESTRATEGIAS TRADUCTOLÓGICAS}

\section{Antropónimos}

Según la estadística de Li, un $63 \%$ de los encabezamientos de esta novela empiezan con antropónimos en ambas estrofas (2012: 327). Tal y como hemos mencionado al principio de este artículo, hay cinco personajes principales en esta novela: el monje, el mono, el cerdo, el bonzo Sha y el caballo. Cada uno de ellos posee diversas denominaciones, tratamientos, alias y nombres metonímicos. El uso de uno u otro en los encabezamientos desvela el cambio de identidad y estado psicológico de los personajes. Por lo tanto, dicha singularidad está íntimamente relacionada con el desarrollo del argumento.

Una vez analizados todos los títulos, hemos de constatar que existen seis denominaciones diferentes para aludir al mono. Utilizamos la herramienta SketchEngine para examinar la concordancia paralela.

Entre todos estos títulos, el Mono-Alma (心猿, xīnyuán) es el que aparece con más frecuencia. Se trata de una palabra que se asimiló al chino a través de la traducción de los sutras. Se utiliza como un símil para referirse al estado de ánimo de una persona en el que los pensamientos se le ocurren sin cesar, como los movimientos constantes del mono. Describe a la gente a quien resulta difícil aspirar a una armonía interior $(\mathrm{Hu}$, 2003: 206). Por ello, el autor adopta este tratamiento para referirse al Mono en los casos en que éste ostenta su poder mágico o pone de manifiesto, a libero arbitrio, su temperamento. Los traductores de ambas versiones lo transfieren, en la mayoría de los casos, de una manera literal sin explicar sus connotaciones.

El Gran-Sabio, el Rey de los monos y el Sosia del Cielo (大圣, dàshèn) son tratamientos de gran prestigio, mientras que bimawen (顼马温, bìmǎwēn) es un cargo insignificante cuya responsabilidad se limita a cuidar el establo ecuestre del Rey Celestial y, en ambas versiones, se traslada adoptando el pinyin. Con respecto a su otro tratamiento, 行者 (xíngzhě), en TM1 se traduce como Novicio, mientras que en TM2, se emplea peregrino. Según la RAE, novicio se refiere a «la persona que, en la religión donde tomó el hábito, no ha profesado todavía», y peregrino significa que «está en esta vida mortal de paso para la eterna». La voz original en chino es un término budista. Describe a una persona que observa estrictamente el hábito del budismo, pero que todavía no lo ha profesado y, concretamente, lleva una vida austera, viaja y pide limosna para subsistir (Hu, 2003: 207). En términos generales, la 
276 palabra novicio encaja mejor el contexto original, a pesar de que un lector hispanohablante pueda necesitar un paratexto para conocer, por completo, la connotación de esta palabra en el budismo.

Por último, el Metal o el Señor Metal (金公, jingōng) es un tratamiento en el contexto taoísta. Los cinco personajes corresponden a los cinco elementos básicos según dicha religión, los cuales son el metal, la madera, el agua, el fuego y la tierra. En la novela, el mono corresponde al metal, el cerdo a la madera, el bonzo Sha a la tierra, el monje al fuego y el caballo al agua. Este planteamiento no es aleatorio, sino que responde a las relaciones interactivas de los cinco elementos, entre las que cabe destacar la existente entre el metal y la madera, puesto que el metal contradice a la madera según la teoría del taoísmo (Zhang, 2009: 91-94). Ello simboliza las relaciones entre el mono y el cerdo que discuten a menudo. De igual modo, cabe destacar que en tales disputas el mono siempre vence al cerdo en última instancia.

En definitiva, los tratamientos personales desempeñan múltiples funciones. No solo desvelan el cambio de identidad y el estado de ánimo de los personajes, sino que también aclaran las relaciones entre ellos y, por ende, transfieren diferentes prosodias semánticas a los lectores. En cuanto a las traslaciones, los autores de ambas versiones respetan, en la mayoría de los casos, las diferencias en los llamamientos, recurriendo al uso del pinyin o a la estrategia de extranjerización para realizar una traducción literal.

\section{Términos taoístas y budistas}

Otro fenómeno al que también hemos aludido con anterioridad es la presencia frecuente de términos propios del budismo y del taoísmo, los cuales indican lugares, personajes, objetos o conceptos relacionados con ambas religiones.
Con respecto a los budistas, los traductores de TM2 emplean más el recurso del sánscrito latinizado que los de TM1. Sin embargo, este fenómeno no puede generalizarse si se tiene en cuenta el caso de la traducción de «袈乷》 $(j i \bar{a} s h \bar{a})$, que en TM1 se traduce por el sánscrito latinizado Kasa$y a$, mientras que en TM2 se opta por túnica que es un vocablo no necesariamente budista. En cuanto a la traducción de 《菩提》 (pútî), que es una transcripción de la palabra Bodhi en sánscrito, se traduce en TM1, a veces, como ilustración, y otras veces, como sabiduría absoluta, mientras que en TM2 como Bodhi. Aunque para un lector hispanohablante, traducciones domesticadas como las de TM1 resultan más fáciles en comprender, la palabra ilustración puede provocar ambigüedad al confundirse con su significado moderno.

Los términos taoístas son más originales, puesto que se trata de una ideología derivada y enraizada en la cultura china y, por ello, provocan más problemas tanto a la hora de distinguir los términos de las palabras, como en su versión. El ejemplo más representativo es la traducción de 《刀圭» $(d \bar{a}$ ogu $\vec{\imath})$. Originalmente, esta palabra describe una herramienta de medida de la medicina tradicional china; posteriormente, también se usó para referirse a una píldora y a la tierra, uno de los cinco elementos básicos, que representa al bonzo Sha (Guo, 1997: 64-69). En TM1 esta voz se traduce como cuchillo, que se aleja en gran medida del significado original, mientras que en TM2 se elimina su traducción.

Por otro lado, la palabra «贞儿» (ying'ér) parece un vocablo bastante común en el lenguaje cotidiano, que significa bebé. No obstante, en la cultura taoísta es un término que se refiere al plomo, pero también significa volver a nacer o $r$ encarnación, ya que, según los taoístas, la infancia es la etapa más vigorosa de nuestra existencia (Zhong, 2001: 211). En la novela se utiliza esta palabra/término para indicar a los niños, pero 
al mismo tiempo alude aparentemente a dicha tradición oriental. En TM1 y TM2, se vierte como niño, muchacho o hijo, dependiendo del contexto. Por lo tanto, las translaciones solo transfieren parcialmente el significado original filtrando su dimensión religiosa. Como norma general, los traductores de ambas versiones adoptan la versión literal para tratar con los términos taoístas.

\section{Numerales}

Los numerales en mandarín son, de igual manera, difíciles de verter. Solían colocarse dos para implicar el resultado de la multiplicación en los textos antiguos chinos. Pongamos el título del capítulo 99 a modo de ejemplo:

Ejemplo 12) TO (Capítulo 99): 九九/数完/ 魔灭尽//三三/行满/道归根//

Transcripción en pinyin: jiujiu/shuwan/mo miejin//sansan/xingman/dao guigen//

TM1: Cuando se completan los nueve nueves, desaparecen todos los demonios. Tras el cumplimiento del triple tres, el dao retorna a su origen.

TM2: NUEVE VECES NUEVE ES EL NÚMERO PERFECTO PARA LOGRAR LA TOTAL EXTINCIÓN DE MARÁ. EL TAO REVIERTE A SUS ORÍGENES, UNA VEZ CONCLUIDOS LOS TRABAJOS DEL TRES DOBLE. [nota: el término «san-san» («tres doble» 0 «tres veces tres» hace referencia a los temas centrales de la meditación budista: el «kung», por el que la mente se ve libre de todo pensamiento; el «wu-hsiang», por el que se desconecta de cualquier fenómeno externo; el «wu-yüan», por el que se libera de las ataduras del deseo. Su forma reduplicativa alude a un nivel superior de meditación. En un sentido taoísta, no obstante, se refiere al proceso completo de la alquimia interna, al relacionar los sesenta y ocho hexagramas del I Ching con los diferentes momentos de las fases lunares.]

Los dos primeros caracteres de la primera oración son, literalmente, nueve nueve, que indican el valor de la multiplicación (a saber: 81). Se trata del número exacto de los problemas que encuentran y solucionan los personajes de la obra al concluir su viaje. Los dos primeros de la segunda mitad del título son, literalmente, tres tres, que se refiere a las tres encarnaciones en vida (Mi y Muñoz, 2020: 290). En TM1 se traducen como «los nueve nueves» $\mathrm{y}$ «triple tres» respectivamente $\mathrm{y}$, en TM2 «NUEVE VECES NUEVE» $\mathrm{y}$ «EL TRES DOBLE», más una nota detallada en la que explican sus significados en los contextos budista y taoísta. De esta manera, los traductores de ambas versiones acuden a la estrategia de la extranjerización, respetando más la estructura formal y la connotación religiosa de los numerales.

\section{CONCLUSIÓN}

En este artículo, hemos explorado, en primer lugar, las características principales de los cien títulos de los capítulos de Viaje al Oeste, una de las novelas clásicas más representativas de China, que marca el principio de la época de madurez en lo que se refiere a la creación estilística en este tipo de encabezamientos (Li, 2012: 190). Sintácticamente, los títulos muestran un paralelismo formado por cuatro, cinco, siete u ocho caracteres en cada estrofa con ritmo y métrica y, en la mayoría de los casos, conjuntamente con rima y juego de combinación de tonos. Léxicamente, contienen una variedad de elementos culturales como los nombres metonímicos de personajes, los términos budistas y taoístas y los numerales, entre otros. Además, estos títulos narrativos 
278 forman prolepsis que explican de antemano los sucesos de cada capítulo. Por todo ello, tanto la forma expresiva, como el contenido y las connotaciones religiosas y de valor configuran las particularidades de estos encabezamientos.

Con respecto a las características generales de las dos trasladaciones de los títulos de capítulos de la dicha novela, los traductores de TM2 suelen construir oraciones más largas (con una mayor carga semántica), mientras que los de TM1 adoptan palabras más variables y complejas. En general, ambas versiones pierden la simetría sintáctica, léxica y rítmica que crea a conciencia el autor en el texto origen.

Mediante un análisis comparativo entre el TO y sus dos traducciones, hemos constatado que el paralelismo sintáctico de los títulos puede servir de referencia que favorece la comprensión del contenido e impide la aparición de malentendidos. Con respecto a las frases que contienen serialización verbal, se deben distinguir las relaciones entre las dos acciones que tienen lugar (según se desarrolla la novela) para representarlas con exactitud y transformarlas con métodos gramaticales tales como la utilización de la conjunción en el texto meta. En cuanto al caso de la omisión de sujeto o el uso de un sustantivo abstracto como el sujeto, es factible el empleo de la forma pasiva en español.

En ambas versiones se adopta el sánscrito latinizado para transferir los términos budistas y crear una traducción más literal (en la mayoría de los casos) para tratar con los términos taoístas. Así mismo, por lo conciso que es el original y el uso abundante de términos religiosos, se opta, en ciertos casos, por la eliminación de elementos (en particular, los que provocan malinterpretaciones) en los dos textos meta.

Por medio de la comparación entre los TM1/2 y el TO, se pueden concluir reglas generales y estrategias concretas de traslación de los títulos de capítulos de novelas clásicas chinas y, al mismo tiempo, detectar ciertos errores evidentes que se deben evitar en futuras prácticas.

Finalmente, y debido a lo reducido del corpus, los valores de los datos solo reflejan las características de traducción de los títulos de los capítulos de la novela Viaje al Oeste y un panorama más completo de estas particularidades requiere la construcción de un corpus de mayores dimensiones y una investigación más exhaustiva.

\section{REFERENCIAS}

Anónimo (2006): Viaje al Oeste: las aventuras del Rey Mono, trad. E. P. Gatón y I. Huang-Wang, Madrid: Siruela.

CORPAS PAstoR, Gloria (2008): Investigar con corpus en traducción: los retos de un nuevo paradigma, Fráncfort: Peter Lang.

GarcíA YeBra, Valentín (1997): «La voz pasiva francesa y su traducción al español», Thélème. Revista Complutense de Estudios Franceses, 11, 347-353.

GILI GAYA, Samuel (1961): Curso superior de sintaxis española. Barcelona: Biblograf.

Guo, MingZhi (郭明志) (1997): «Daogui yu Xi You Ji renwude bieming daichen» (《刀圭与《西游记》 人物的别名代称》)[《Daogui y los alias y nombres metonímicos en Viaje al Oeste»], Seeking Truth, 2, 64-69.

Hernando Cuadrado, Luis Alberto (2005): «El orden de palabras en español», Revista de filología, 23, 161178.

Hu, Sheng (胡胜) (2003):Xi You Ji cidian (《西游记》 辞典) [Diccionario del Viaje al Oeste], Shenyang: Liaohai.

LAviosA, Sara (2002): Corpus-Based Translation Studies: Theory, Findings, Applications, Ámsterdam: Rodopi.

LI, Xiaolong (李小龙) (2012): Zhonguo gudianxiaoshuo huimu yanjiu (中国古典小说回目研究) [Estudios sobre los títulos de capítulo de las novelas clásicas chinas], Pekín: Peking University Press.

Liu, Junbo (刘骏勃) (2018): «Yijiu kuiren youyanlaizhanghuitixiaoshuode shijuhua biange» (依旧窥 人有燕来一一章回体小说回目的诗句化变革) [«Las golondrinas siempre volverán: revolución de las formas poéticas de los títulos de capítulos en 
las novelas clásicas chinas»], Tangdu Journal, 34/6, 78-84.

Ku, Menghsuan (2019): «Viaje al Oeste vs. viaje a la diversión: estrategias de traducción de los elementos culturales de Peregrinación al Oeste». Onomázein: Revista de lingüística, filología y traducción de la Pontificia Universidad Católica de Chile, 43: 50-69.

McEnery, Tony y Andrew Hardie (2012): Corpus Linguistics: Method, Theory and Practice, Cambridge: Cambridge University Press.

Mi, Tian y Rodrigo MuÑoz Cabrera (2020): «¿Numerales que atraviesan la barrera lingüística? Un estudio práctico de la traducción del 9 del chino al español por medio del Corpus CCEVAO». Estudios de Traducción, 10, 285-298.

Munday, Jeremy (2001): Introducing Translation Studies: Theories and Applications, Londres-Nueva York: Routledge.

Nemark, Peter (2002): A Textbook of Translation, Shanghái: Shanghai Foreign Language Education Press.

NiDA, Eugene A. (2017): Language and Culture. Contexts in Translating, Shanghai: Shanghai Foreign Language Education Press.

Nord, Christiane (1990): «Funcionalismo y lealtad: algunas consideraciones en torno a la traducción de títulos», en Margit Raders y Juan Conesa (1990), II Encuentros Complutenses en torno a la traducción. Madrid: Editorial Complutense. 153-162.

Real Academia Española (2014): Diccionario de la lengua española, Madrid: Espasa.

Real Academia Española (1999): Ortografía de la lengua española, Madrid: Espasa.

Tognini-Bonelli, Elena (2001): Corpus Linguistics at Work, Ámsterdam, Benjamins.

VenutI, Lawrence (1995): The Translator's Invisibility: A History of Translation. Londres: Routledge.

VIEZZI, Maurizio (2011): «The Translation of Book Titles: theoretical and practical Aspects», en Pekka Kujamäki, Leena Kolehmainen y Hannu Penttilä (eds.), Beyond Borders - Translations Moving Languages, Literatures and Cultures, Berlín: Frank \& Timme, 183-195.
W ANG, Li (王力) (2014): Zhongguo xiandai yufa (中国 现代语法) [Gramática de chino moderno], Beijing: Zhonghua Press.

Wu, Cheng'en (2010): Peregrinación al Oeste, trad. M. Lacea y C. Trigoso, Beijing: Ediciones en Lenguas Extranjeras.

Wu, Cheng'en (吴承恩) (2018): Xi You Ji (西游记) [Viaje al Oeste], Beijing: Literatura del Pueblo.

ZHANG, Pingren (张平仁) (2009): «Xi You Ji wuxing sixiang pingxi» (《西游记》五行思想评析) [«Crítica de los pensamientos de cinco elementos en Viaje al oeste»], The Journal of Ming-Qing Fiction Studies, 91, 91-94.

ZHENG, Zhenduo (郑振铎) (2012): Zhongguo suwenxueshi (中国俗文学史) [Historia de la literatura vulgar de China], Beijing: Oriental.

ZHoNG, Zhaopeng (钟肇鹏) (2001): Daojiao xiaocidian (道教小辞典) [Diccionario del Taoísmo], Shanghái: Shanghai Lexicographic Publishing House. 